2010-01-01

\title{
A Voltage Sensor Based on a Singlemode-Multimode-Singlemode Fiber Structure
}

\author{
Agus Hatta \\ Technological University Dublin, ahatta@tudublin.ie \\ Yuliya Semenova \\ Technological University Dublin, yuliya.semenova@tudublin.ie \\ Ginu Rajan \\ Technological University Dublin, ginu.rajan@tudublin.ie
}

See next page for additional authors

Follow this and additional works at: https://arrow.tudublin.ie/engscheceart

Part of the Electrical and Computer Engineering Commons

\section{Recommended Citation \\ Hatta, A. et al. (2010) A Voltage Sensor Based on a Singlemode-Multimode-Singlemode Fiber Structure. Microwave and Optical Technology Letters, Vol.52, no.8, pp.1887-1890. doi:10.1002/mop.25339}

This Article is brought to you for free and open access by the School of Electrical and Electronic Engineering at ARROW@TU Dublin. It has been accepted for inclusion in Articles by an authorized administrator of ARROW@TU Dublin. For more information, please contact arrow.admin@tudublin.ie, aisling.coyne@tudublin.ie, gerard.connolly@tudublin.ie.

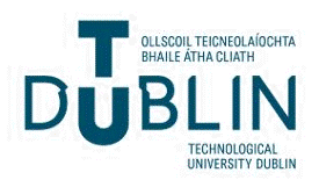




\section{Authors}

Agus Hatta, Yuliya Semenova, Ginu Rajan, and Gerald Farrell

This article is available at ARROW@TU Dublin: https://arrow.tudublin.ie/engscheceart/69 


\title{
A voltage sensor based on a Singlemode- Multimode-Singlemode fiber structure
}

\author{
A. M. Hatta, Y. Semenova, G. Rajan, and G. Farrell
}

\begin{abstract}
A voltage sensor based on a singlemode-multimodesinglemode (SMS) fiber structure, attached to a piezoelectric $(\mathrm{PZT})$, utilized in a ratiometric optical power measurement scheme is proposed. The elongation of the PZT due to an applied voltage induces a strain on the SMS fiber structure and in turn results in a change in the transmission loss response of the SMS fiber structure. It is found that at selected wavelengths, a strong voltage dependence for the transmission loss of the SMS fiber structure can be achieved. The voltage information can be obtained from the power ratio of the system, assuming the system is calibrated. The proposed voltage sensor offers a simpler configuration when compared to other fiber optic voltage sensors.
\end{abstract}

Index Terms-fiber optic sensor, voltage measurement

\section{INTRODUCTION}

$\bigcirc$ ptical fiber voltage sensors have a number of advantages over conventional voltage sensors such as resistance to electromagnetic interference, low weight, compactness, fast response, and are also suitable for remote operation [1]. In some optical fiber voltage sensors, piezoelectric (PZT) devices have been utilized as transducers. The optical fiber sensor is securely attached to the PZT so that a voltage applied to the PZT induces a strain in the PZT and thus in the optical fiber sensor. By using the fiber to sense the strain, voltage can be measured. The fiber sensor could be a singlemode fiber [2], a hollow fiber [3], or a fiber-Bragg grating (FBG) [4]. Depending on the fiber sensor used, the measurement of strain and thus voltage can be carried out using an interferometeric measurement system [2, 3] or by measuring reflected wavelength [4]. Both of these measurement schemes are complex and expensive to implement.

A singlemode-multimode-singlemode (SMS) fiber structure has been used to sense strain [5]. The characteristics of the wavelength shift of the SMS spectral response due to the strain applied to SMS fiber structure have been investigated previously in $[5,6]$. In this paper, by utilizing the effect of strain on the SMS fiber structure, we present for the first time a simple method to measure DC voltage using a SMS fiber

Manuscript received October 12, 2009.

A. M. Hatta*, Y. Semenova, G. Rajan, and G. Farrell, are with Photonics Research Centre, Dublin Institute of Technology, Dublin, Ireland (e-mail: ahatta@dit.ie).*On leave from Engineering Physics Department, Institut Teknologi Sepuluh Nopember (ITS), Surabaya, Indonesia. structure attached to a PZT stack, while employing a simple ratiometric power measurement scheme. The specific advantages of utilizing a SMS fiber structure over the FBG or hollow fiber PZT-based voltage sensors are ease of fabrication, low cost and simplicity of interrogation. A DC voltage sensor is demonstrated with a range of 0 to $100 \mathrm{~V}$ which is suitable for remote voltage monitoring. The sensor is connected to the measurement system only by fiber and thus has the advantages that the sensor requires no local electrical power source and is also electrically isolated. Possible applications include voltage monitoring in areas with high electrical field strengths. Moreover it has the potential to be applied to high voltage sensor applications with a suitable choice of PZT.

\section{STRAIN DEPENDENCE OF SMS FIBER STRUCTURE}

Strain and consequent elongation is produced in a PZT when a voltage is applied. The voltage sensor is fabricated by securely bonding a SMS fiber structure to the PZT stack, as shown in Fig. 1. Two ends of the PZT stack are bonded between a solid fixed platform and a light weight bracket. The two ends of the SMS fiber structure are glued to the platform and the bracket. The elongation of the PZT, when voltage is applied, will induce an axial strain in the SMS fiber structure. It is shown in $[5,6]$ that the spectral response of a SMS fiber structure shifts to a lower wavelength due to applied strain. As a result if the transmission loss of the SMS fiber structure is

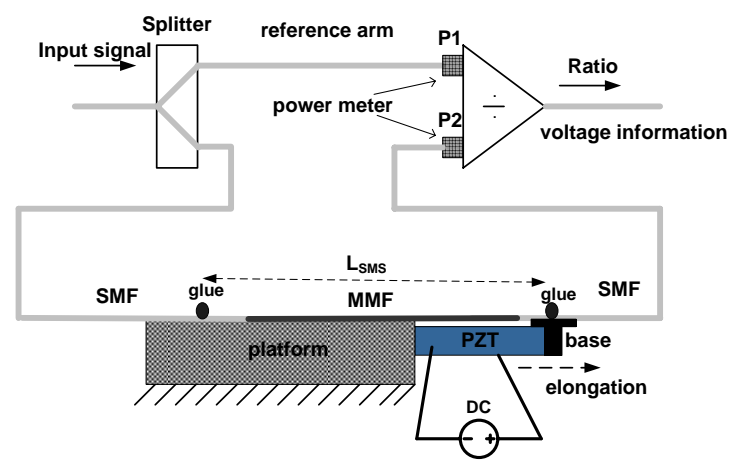

Fig. 1. Schematic configuration of SMS fiber structure voltage sensor system.

measured at a fixed wavelength, the loss will be proportional to the strain and thus the voltage.

Fig. 1 shows a schematic configuration of the voltage sensor using a ratiometric power measurement system. The optical input signal is divided into two equal power signals, one goes to the reference arm and the other goes to the SMS 
fiber structure. Two photodiodes and associated electronics are used to measure the optical output power ratio from the two arms. A ratiometric scheme is used to ensure independence from input signal power variations improving the stability and accuracy of the measurement system. An unknown applied voltage can be measured by measuring the ratio of the photodiode outputs, assuming the system is calibrated.

To optimize a SMS fiber structure and to select an operating wavelength, it is useful to initially investigate the strain dependence of the spectral response of a SMS fiber structure. A modal propagation analysis for linearly polarized (LP) modes can be used to investigate light propagation in the SMS fiber structure [7]. It is known that an applied strain $(\varepsilon)$ induces a change in the length $(\Delta L)$ and the core radius $(\Delta a)$ of a SMS fiber structure employing a step index MMF and also change the refractive index $(\Delta n)$ of the SMF and MMF sections. For an applied strain $(\varepsilon)$, the change in MMF length, the core radius and refractive index can be expressed as [6]:

$$
\begin{gathered}
\Delta L=L \varepsilon \\
\Delta a=-v a \varepsilon \\
\Delta n_{i}=-\frac{n_{i}^{2}}{2}\left[p_{12}-v\left(p_{11}+p_{12}\right)\right] \varepsilon=-p_{e} \varepsilon
\end{gathered}
$$

where $L$ is the length of MMF, $a$ is the core radius of SMF/MMF, $n_{i}$ represents the refractive index of the core and cladding of the SMF/MMF, $p_{11}$ and $p_{12}$ are strain-optic coefficients for fused silica, $p_{e}$ is the effective strain-optic coefficient, and $v$ is the Poisson ratio.

In this paper, the core/cladding diameter of the SMF is 9/125 $\mu \mathrm{m}$ and of the MMF is $105 / 125 \mu \mathrm{m}$, while an MMF length of $43.93 \mathrm{~mm}$ are used for calculation. MMF lengths from 44.19 to 41.54 provide a bandpass spectral response with a peak bandpass wavelength from 1500 to $1600 \mathrm{~nm}$, respectively [7]. The spectral response of the SMS fiber structure is calculated using the MPA and it is shown in Fig. 2 for the case without an applied strain, $\Delta \varepsilon=0 \mu \varepsilon$. The bandpass spectral response is shown with the peak bandpass wavelength of $1510 \mathrm{~nm}$.

To determine which wavelength of the response is most sensitive to strain, a strain dependent loss (SDL) of the response is investigated. The SDL is defined as the transmission loss difference between with- and withoutapplied strain. A commercial PZT stack AE0505D18 (from Thorlabs) is used as the transducer. It has a length of $20 \mathrm{~mm}$ and cross sectional dimensions of $5 \times 5 \mathrm{~mm}$. The maximum elongation is $15 \mu \mathrm{m}$ at a maximum DC voltage of $100 \mathrm{~V}$. For the $\mathrm{L}_{\mathrm{SMS}}=50 \mathrm{~mm}$, the SDL due to the maximum elongation which corresponds to $\Delta \varepsilon=300 \mu \varepsilon$ is calculated. The strain related coefficient of $p_{e}=0.22$ and $v=0.16$ are used as in [6]. In Fig. 2, the calculated spectral response is shown at $\Delta \varepsilon$ $=300 \mu \varepsilon$, while the inset figure in Fig. 2 shows the SDL of SMS fiber structure. It is clear that at certain wavelengths, the sensitivity to strain is much higher and such wavelengths are suitable operating wavelengths for voltage measurement. A wavelength of $1556.8 \mathrm{~nm}$, is a good example of a suitable wavelength due to its high SDL value which will improve strain sensitivity and thus voltage sensitivity. In Fig. 3, the transmission loss response, at $1556.8 \mathrm{~nm}$, due to the applied

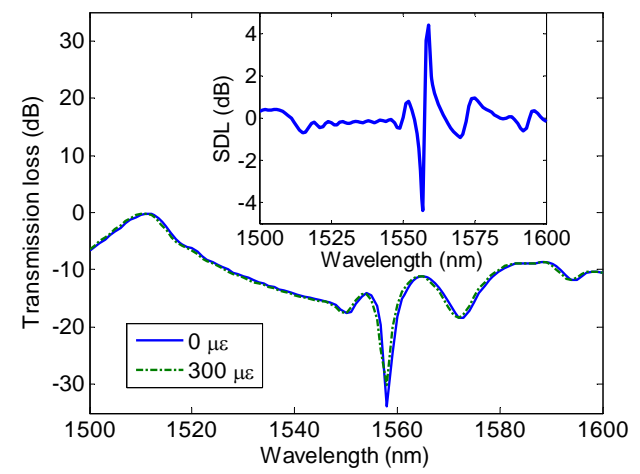

Fig. 2. Transmission loss response of the SMS fiber structure. SDL of the SMS fiber structure (inset figure).

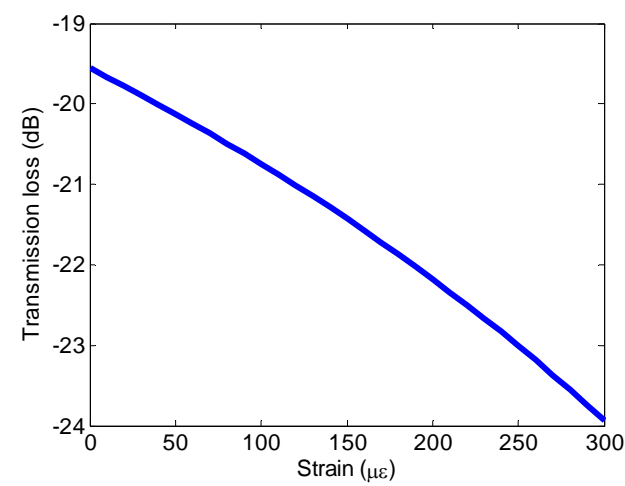

Fig. 3. Transmission loss vs applied strain to the SMS fiber structure at a wavelength of $1556.8 \mathrm{~nm}$.

strain from 0 to $300 \mu \varepsilon$ is shown with a monotonically decreasing response and a discrimination range of $-4.378 \mathrm{~dB}$. This is equivalent to a strain sensitivity of $0.0146 \mathrm{~dB} / \mu \varepsilon$ and thus a voltage sensitivity of $0.0438 \mathrm{~dB} / \mathrm{V}$.

\section{EXPERIMENTAL RESULTS}

The SMS fiber structure described above was fabricated using a Fujikura CT-07 cleaver and a Sumitomo type-36 fusion splicer. As shown in Fig. 1, the SMS fiber structure is glued to the platform and the bracket at two fixed points, with a total length between the fixed points of about $50 \mathrm{~mm}$. The PZT stack AE0505D18 was used as the transducer.

A tunable laser TUNICS-PLUS was used as the input signal source. Initially to select the appropriate wavelength, two spectral ratio responses were measured at voltages of 0 and 100 V. A single channel piezo controller MDT694A (Thorlabs) was used to supply DC voltages from 0 to $100 \mathrm{~V}$. The measured spectral ratio responses at $0 \mathrm{~V}$ and $100 \mathrm{~V}$ for a wavelength range of 1500 to $1600 \mathrm{~nm}$ with an increment of $0.1 \mathrm{~nm}$ are shown in Fig. 4. It can be seen that the spectral response of the SMS fiber structure is shifted to a lower 
wavelength region when the voltage is applied. This spectral response shift to the lower wavelength region confirms the strain effect on the SMS fiber structure as in [5, 6].

For the purpose of ratiometric power based measurement, it is necessary to select the optimal operating wavelength. The ratio difference at $100 \mathrm{~V}$ and $0 \mathrm{~V}$ is presented in the inset in Fig. 4. A wavelength of $1556.8 \mathrm{~nm}$ shows the largest ratio difference of $-1.95 \mathrm{~dB}$ between $0 \mathrm{~V}$ and $100 \mathrm{~V}$ and thus is selected as the operational wavelength. The measured ratio response due to an applied voltage from 0 to $100 \mathrm{~V}$ with an increment of $5 \mathrm{~V}$ at the operational wavelength of $1556.8 \mathrm{~nm}$ is presented in Fig. 5. A monotonic decrease in the ratio response with applied voltage is demonstrated with a sensitivity of $0.0195 \mathrm{~dB} / \mathrm{V}$. The experimental sensitivity is lower than that predicted but this discrepancy can be

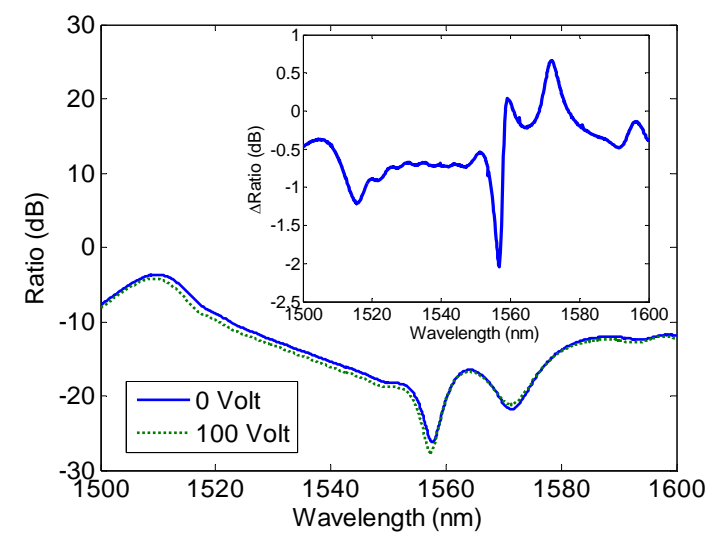

Fig. 4. Measured ratio spectral response at $0 \mathrm{~V}$ and $100 \mathrm{~V}$ (inset: ratio difference)

attributed to small length errors in the fabricated SMS structure and the accuracy of the coefficient strain values used in the calculations. Assuming a power measurement resolution of $0.005 \mathrm{~dB}$ is possible, then the voltage measurement resolution should be better than $0.5 \mathrm{~V}$.

To check the voltage measurement resolution of the system, a step change of $0.5 \mathrm{~V}$ from 50 to $52 \mathrm{~V}$, with a time interval of 5 seconds is applied to the SMS fiber structure sensor. The measured ratio variation is shown in the inset of Fig. 5 . It is clear that the resolution of the voltage sensing system is better than $0.5 \mathrm{~V}$, confirming that the SMS fiber structure, together with a PZT, is suitable as a voltage sensor for DC voltage measurements from 0 to 100 volts.

It is well known that the PZT exhibits the hysteresis in strain to voltage response and therefore we also observed the hysteresis in measured ratio response against the voltage. However hysteresis can be minimized by the selection of an appropriate PZT material [8] or by applying numerical techniques to compensate for the hysteresis [9].

For a given fixed signal source wavelength, the MMF length will need to be optimized in order to achieve the highest ratio (and thus voltage) dependence. It is shown in [7], that the peak wavelength of a bandpass filter can be tuned by selecting a suitable MMF length. It is also possible to use the SMS fiber structure for high voltage measurements. The operating voltage depends on the PZT's strain-voltage characteristic. In [4], a FBG is attached to a PZT as a high voltage sensor. The PZT in [4] can be operated over a voltage range from 0 to $5 \mathrm{kV}$ with a maximum PZT elongation of

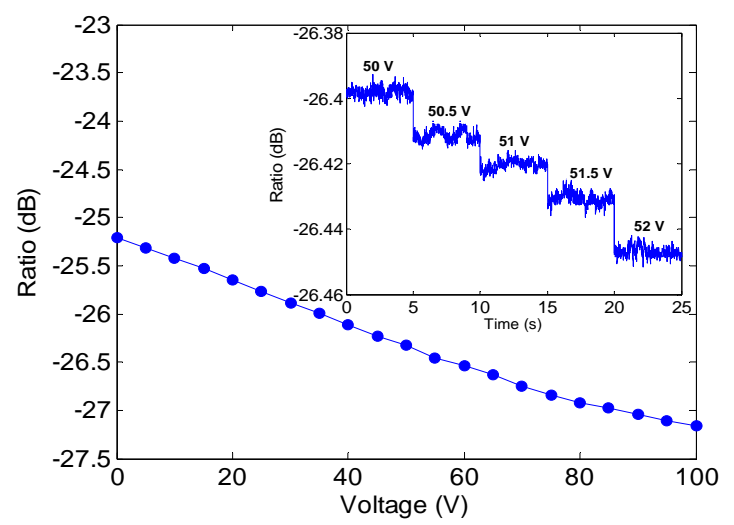

Fig. 5. Ratio response of system against voltage at the operational wavelength $1556.8 \mathrm{~nm}$ (inset: variation in ratio for a step change of $0.5 \mathrm{~V}$ ) about $19 \mu \mathrm{m}$ which is similar to the maximum PZT elongation used in this paper. The maximum PZT elongation should be within the strength limits of the SMS fiber structure, which is approximately $100 \mu \mathrm{m}$ in the present case.

\section{CONCLUSION}

The SMS fiber structure attached to the PZT transducer is proposed as a voltage sensor employed in a ratiometric power measurement scheme. A DC voltage sensor from 0 to $100 \mathrm{~V}$ with a resolution about $0.5 \mathrm{~V}$ is demonstrated. The proposed sensor requires no electrical power source and is electrically isolated. It utilizes a simple ratiometric power measurement system, offering a fast measurement capability with the potential for kilovolt measurement with a suitable choice of PZT.

\section{REFERENCES}

[1] G. Fusiek, P. Niewczas and J. R. McDonald, ”Feasibility study of the application of optical voltage and current sensors and an arrayed waveguide grating for aero-electrical systems," Sens. and Actuat. A, Vol. 147, pp. 177-182, 2008.

[2] L. M-Leom, A. DIez, J. L. Cruz and M. V. Andres, "Frequency-output fiber-optic voltage sensor for high-voltage lines,” IEEE Phot. Technol. Lett., Vol. 13, No. 9, pp. 996-998, 2001.

[3] S. Kim, J. Park and W.-T. Han, "Optical fiber AC voltage sensor," Microwave and Opt. Technol. Lett., Vol. 51, No. 7, pp. 1689-1691, 2009.

[4] P. Niewczas, L. Dziuda, G. Fusiek and J. R. McDonald, "Design and evaluation of a preprototype hybrid fiber-optic voltage sensor for a remotely interrogated condition monitoring system,” IEEE Trans. On Inst. and Meas., Vol. 54, No. 4, pp. 1560-1564, 2005

[5] S. M. Tripathi, A. Kumar, R. K. Varshney, Y. B. P. Kumar, E. Marin and J. P. Meunier, "Strain and temperature sensing characteristics of single-mode-multimode-single-mode structures,” J. Lightwav. Technol., Vol. 27, No. 13, pp. 2348-2356, 2009.

[6] E. Li, "Temperature compensation of multimode interference-based fiber devices,” Opt. Lett. Vol. 32, No. 14, pp. 2064-2066, 2007.

[7] Q. Wang, G. Farrell, and W. Yan, "Investigation on singlemodemultimode-singlemode fiber structure,” J. Lightwave Technol., Vol. 26, No. 5, pp. 512-519, 2008.

[8] M. Pacheco, F. M. Santoyo, A Mendez, and L. A Zenteno, "Piezoelectric-modulated optical fibre Bragg grating high-voltage sensor,” Meas. Sci. Technol., Vol. 10, pp. 777-782., 1999. 
[9] G. Fusiek, P. Niewczas, L. Dziuda, and J. R. McDonald, "Hysteresis compensation for a piezoelectric fiber optic voltage sensor," Opt. Eng., Vol. 44, No. 11, pp.114402, 2005. 